\title{
Quasilinearization for nonlinear boundary value problems for delay-type difference equations with maxima
}

\author{
Snezhana Hristova* and Angel Golev
}

\section{"Correspondence:}

snehri@uni-plovdiv.bg

Plovdiv University, Tzar Asen 24,

Plovdiv, 4000, Bulgaria

\begin{abstract}
The paper deals with an approximate method for solving a mixed boundary value problem for nonlinear difference equations containing a maximum of the unknown function over a past time interval. Every successive approximation to the unknown solution is the unique solution of an appropriately constructed initial value problem for a linear difference equation with maxima, and an algorithm for its explicit obtaining is suggested. Also, each approximation is a lower/upper solution of the given mixed problem. The rapid convergence of the successive approximations is proved. The suggested algorithm is realized as a computer program and it is applied to an example.
\end{abstract}

MSC: 39A23; 39A99; 65Q10

Keywords: delay-difference equations with maxima; boundary value problem; approximate solution; computer realization

\section{Introduction}

In the last few decades great attention has been paid to automatic control systems and their applications to computational mathematics and modeling. Many problems in the control theory correspond to the maximal deviation of the regulated quantity. Discrete modeling of such kind of problems is done adequately by difference equations with maxima over a past time discrete interval. These difference equations are a part of the set of difference equations with delays. Meanwhile, the delays have recently been found crucial in many areas such as neuronal dynamics. For example, the effects of periodic subthreshold pacemaker activity and time-delayed coupling on stochastic resonance over scale-free neuronal networks are studied in [1]; the effects of spatiotemporal additive noise on the spatial dynamics of excitable neuronal media that is locally modeled by a two-dimensional map are considered in [2]; the discrete model of the movement of eukaryotic cells regulated by a process of phase separation of two competing enzymes on the cell membrane is studied in [3]; front propagation and synchronization transitions in dependence on the information transmission delay and coupling strength over scale-free neuronal networks with different average degrees and scaling exponents are investigated in [4].

The presence of the maximum function over a discrete past time interval in the discrete equation requires not only more complicated calculations but also a development of new methods for qualitative investigations of the behavior of their solutions as well as

○2014 Hristova and Golev; licensee Springer. This is an Open Access article distributed under the terms of the Creative Commons Attribution License (http://creativecommons.org/licenses/by/2.0), which permits unrestricted use, distribution, and reproduction in any medium, provided the original work is properly cited. 
approximate methods for their solving. The character of the maximum function leads to a variety of different types of difference equations. The properties of solutions of some special types of difference equations with maxima are studied in [5-7]. In several papers various types of boundary value problems for difference equations have been studied and the monotone iterative method has been applied. For example, in $[8,9]$ first-order difference equations are studied; in [10] some criteria for existence and uniqueness results for nth order anti-periodic difference equations are developed; in [11] a generalized delay difference equation is studied by lower and upper solutions, but the problem consists only of a boundary condition, which does not get uniqueness of the solution; the global boundary value problem for difference equations without any kind of delay is well studied in [12]; in [13] a nonlinear boundary value problem for a delay difference equation with one delay is studied by the monotone iterative method; in [14] an approximate method with a rapid convergence is applied to an initial value problem for difference equations with maxima. Also, in [15], the monotone iterative technique is applied to a periodic boundary value problem for difference equations with maxima, but the successive approximation is solutions of periodic boundary value problems, which are practically difficult to be obtained. Approximate methods for various problems for differential equations with maxima are proved and applied in [16-18].

In the paper a nonlinear difference equation of delayed type is considered. The studied equation generalizes the well-known problems in several ways:

- at each current time the value of unknown function is included in both parts of the equation, so the equation could not be solved recursively;

- the delays are without any restrictions;

- the boundary condition is set up in a very general way and it involves many other cases, which are studied in the literature (see, for example, the above-mentioned papers).

The main purpose of the paper is to establish comparison results which allow us to use upper and lower solutions in order to build two convergent monotonic sequences of functions with discrete domains. Each term of these sequences is a solution of an appropriately constructed initial value problem for a linear difference equation with maxima. An algorithm for solving these initial value problems is given. Also, each term of both sequences is a lower/upper solution of the given nonlinear boundary value problem. The limits of both sequences coincide to the solution of a given problem and the rapid convergence of both sequences is proved. Also, the algorithm is computerized and it is applied to a particular example to show the advantages of the suggested approximate method.

\section{Statement of the problem}

Let $\mathbb{R}_{+}=[0, \infty), \mathbb{Z}$ be the set of all integers. For any $c, b \in \mathbb{Z}: c<b$, we denote $\mathbb{Z}[c, b]=\{z \in$ $\mathbb{Z}: c \leq z \leq b\}$.

Let $a, T \in \mathbb{Z}: T>a+1, r, p \in \mathbb{Z}[0, T-a]$ and $h \in \mathbb{Z}: h>0$ be fixed.

Consider the following mixed boundary value problem for a nonlinear delay-difference equation with 'maxima' (MBVP):

$$
\begin{aligned}
& \Delta u(k-1)=f\left(k, u(k), u\left(\tau_{1}(k)\right), u\left(\tau_{2}(k)\right), \ldots, u\left(\tau_{r}(k)\right), \max _{s \in \mathbb{Z}[k-h, k]} u(s)\right) \\
& \quad \text { for } k \in \mathbb{Z}[a+1, T],
\end{aligned}
$$




$$
\begin{aligned}
& u(k)=\varphi(k) \quad \text { for } k \in \mathbb{Z}[a-h+1, a-1], \\
& u(a)=g\left(u\left(T-\lambda_{1}\right), u\left(T-\lambda_{2}\right), \ldots, u\left(T-\lambda_{p}\right), u(T)\right),
\end{aligned}
$$

where $u \in \mathbb{R}, \Delta u(k-1)=u(k)-u(k-1), f: \mathbb{Z}[a+1, T] \times \mathbb{R}^{r+2} \rightarrow \mathbb{R}, g: \mathbb{R}^{p+1} \rightarrow \mathbb{R}, \tau_{m}(k):$ $\mathbb{Z}[a+1, T] \rightarrow \mathbb{Z}[a+1-h, T]: k-h \leq \tau_{m}(k) \leq k, m=1,2, \ldots, r, \lambda_{j} \in \mathbb{Z}[1, T-a], j=1,2, \ldots, p$, and $\varphi: \mathbb{Z}[a-h+1, a-1] \rightarrow \mathbb{R}$.

Any solution of MBVP (1)-(3) is a finite sequence of $T-a+h$ real numbers, and we consider it as a real-valued function with a discrete domain.

The presence of delays $\tau_{m}$ generalizes the type of the considered difference equation since the function $f$ could depend on different delays at any point $k$. In the case $r=h$ and $\tau_{j}(k)=k-j, j=1, \ldots, r$, the right-hand side of (1) is reduced to $f(k, u(k-h), u(k-h+$ $\left.1), \ldots, u(k-1), u(k), \max _{s \in \mathbb{Z}[k-h, k]} u(s)\right)$. For some other particular cases, see equation (39) of the paper.

The presence of both delays and the maximum function in the equation leads to a new statement of the problem, which involves both the boundary condition and the initial condition. Problem (1)-(3) covers many different problems for difference equations with delays and maxima such as the initial value problem, the periodic boundary value problem, the linear boundary value problem.

\section{Preliminary notes, basic notations and definitions}

Note $\sum_{i=n}^{m} a_{i}=0$ and $\prod_{i=n}^{m} a_{i}=1$, where $m<n<\infty$ and $a_{j} \in \mathbb{R}, j \in Z[m, n]$.

For any function $F \in C^{2}(I, \mathbb{R}), I \subset \mathbb{R}^{r+2}, V=\left(v_{1}, v_{2}, \ldots, v_{r+2}\right)$, we denote by $F_{v_{j}}(V)$ the first derivative of $F(V)$ with respect to its $j$ th argument, and by $F_{v_{j} v_{k}}(V)$ the second derivative of $F(V)$ with respect to its $j$ th and $k$ th arguments.

We introduce the following notations:

$$
\begin{aligned}
& \chi(u(T))=\left(u\left(T-\lambda_{1}\right), u\left(T-\lambda_{2}\right), \ldots, u\left(T-\lambda_{p}\right), u(T)\right), \\
& U(u(\tau(k)))=\left(u\left(\tau_{1}(k)\right), u\left(\tau_{2}(k)\right), \ldots, u\left(\tau_{r}(k)\right)\right), \\
& \Xi(u(\tau(k)))=\left(u(k), U\left(u(\tau(k)), \max _{s \in \mathbb{Z}[k-h, k]} u(s)\right)\right), \quad k \in \mathbb{Z}[a+1, T] .
\end{aligned}
$$

Using the above notations, the right-hand sides of equation (1) and boundary condition (3) could be written in a simpler way: $f(k, \Xi(u(\tau(k))))$ and $g(\chi(u(T)))$.

We will use the norm $\|u\|=\max \{|u(k)|: k \in \mathbb{Z}[a-h+1, T]\}$.

Let $\alpha, \beta: \mathbb{Z}[a+1-h, T] \rightarrow \mathbb{R}$ be given functions such that $\alpha(k) \leq \beta(k)$. We introduce the following sets:

$$
\begin{aligned}
& S(\alpha, \beta)=\{u: \mathbb{Z}[a-h+1, T] \rightarrow \mathbb{R}: \alpha(k) \leq u(k) \leq \beta(k), k \in \mathbb{Z}[a-h+1, T]\}, \\
& W(\alpha, \beta)=\left\{x \in \mathbb{R}^{p+1}: \chi(\alpha(T)) \leq x \leq \chi(\beta(T))\right\}, \\
& \Omega_{k}(\alpha, \beta)=\left\{V \in \mathbb{R}^{r+2}: \Xi(\alpha(\tau(k))) \leq V \leq \Xi(\beta(\tau(k)))\right\}, \quad k \in \mathbb{Z}[a+1, T] .
\end{aligned}
$$

We will use lower/upper solutions of MBVP (1)-(3) which are defined in a well-known way. 
Definition 1 [11] The function $\alpha: \mathbb{Z}[a-h+1, T] \rightarrow \mathbb{R}$ is called a lower (upper) solution of MBVP (1)-(3) if

$$
\begin{aligned}
& \Delta \alpha(k-1) \leq(\geq) f\left(k, \alpha(k), \alpha\left(\tau_{1}(k)\right), \alpha\left(\tau_{2}(k)\right), \ldots, \alpha\left(\tau_{r}(k)\right), \max _{s \in \mathbb{Z}[k-h, k]} \alpha(s)\right) \\
& \quad \text { for } k \in \mathbb{Z}[a+1, T], \\
& \alpha(k) \leq(\geq) \varphi(k) \quad \text { for } k \in \mathbb{Z}[a-h+1, a-1], \\
& \alpha(a) \leq(\geq) g\left(\alpha\left(T-\lambda_{1}\right), \alpha\left(T-\lambda_{2}\right), \ldots, \alpha\left(T-\lambda_{p}\right), \alpha(T)\right) .
\end{aligned}
$$

\section{Linear delay difference inequalities with maxima}

We will consider the following linear difference inequality with 'maxima':

$$
\begin{aligned}
\Delta u(k-1) \leq & K+Q(k) u(k)+\sum_{j=1}^{r} C_{j}(k) u\left(\tau_{j}(k)\right) \\
& +q(k) \max _{s \in \mathbb{Z}[k-h, k]} u(s), \quad k \in \mathbb{Z}[a+1, T], \\
u(k) \leq K, \quad k \in \mathbb{Z}[a-h+1, a], &
\end{aligned}
$$

where $K$ is a nonnegative constant.

The inequalities (4) are the base of the main proof, and we will obtain a solution of (4) in an explicit form.

Lemma 1 Let the following conditions be fulfilled:

1. The delays $\tau_{j}: \mathbb{Z}[a+1, T] \rightarrow \mathbb{Z}[a+1-h, T], j \in \mathbb{Z}[1, r]$ are such that $k-h \leq \tau_{j}(k) \leq k$, $k \in \mathbb{Z}[a+1, T]$.

2. The functions $q, Q, C_{j}: \mathbb{Z}[a+1, T] \rightarrow \mathbb{R}_{+}, j \in \mathbb{Z}[1, r]$, and

$$
\tilde{S}(k) \equiv q(k)+Q(k)+\sum_{j=1}^{r} C_{j}(k)<1 \quad \text { for } k \in \mathbb{Z}[a+1, T] .
$$

3. The function $u: \mathbb{Z}[a+1-h, T] \rightarrow \mathbb{R}_{+}$satisfies inequalities (4).

Then for $k \in \mathbb{Z}[a+1, T]$ the inequality $u(k) \leq K \theta(k)$ holds, where

$$
\theta(k)=\frac{1}{\prod_{j=a+1}^{k} 1-\tilde{S}(j)}+\sum_{j=a+1}^{k} \frac{1}{\prod_{\xi=j}^{k} 1-\tilde{S}(\xi)} .
$$

Proof From inequality (4) we obtain

$$
\begin{aligned}
u(k) & \leq \sum_{j=a+1}^{k}\left\{K+Q(j) u(j)+\sum_{i=1}^{r} C_{i}(j) u\left(\tau_{i}(j)\right)+q(j) \max _{s \in \mathbb{Z}[j-h, j]} u(s)\right\}, \\
k & \in \mathbb{Z}[a+1, T] .
\end{aligned}
$$


Define a function $z: \mathbb{Z}[a+1-h, T] \rightarrow \mathbb{R}_{+}$by the equalities

$$
z(k)= \begin{cases}\sum_{j=a+1}^{k}\{K+u(j-1)+Q(j) u(j) & \\ \left.\quad+\sum_{i=1}^{r} C_{i}(k) u\left(\tau_{i}(j)\right)+q(j) \max _{s \in \mathbb{Z}[j-h, j]} u(s)\right\}, & k \in \mathbb{Z}[a+1, T], \\ K, & k \in \mathbb{Z}[a+1-h, a] .\end{cases}
$$

The function $z(k)$ is nondecreasing and $u(k) \leq z(k), u\left(\tau_{j}(k)\right) \leq z\left(\tau_{j}(k)\right) \leq z(k)$, $\max _{s \in \mathbb{Z}[k-h, k]} u(s) \leq \max _{s \in \mathbb{Z}[k-h, k]} z(s)=z(k)$ for $k \in \mathbb{Z}[a+1, T], j=1,2, \ldots, r$.

From the definition of $z(k)$ and the first inequality in (4), we obtain

$$
\left[1-q(k)-Q(k)-\sum_{j=1}^{r} C_{j}(k)\right] z(k) \leq K+z(k-1), \quad k \in \mathbb{Z}[a+1, T] .
$$

By mathematical induction from (8), condition 2 and the definition of $z(k)$, we prove the claim.

In the case when all delays $\tau_{j}(k)<k$ for $k \in \mathbb{Z}[a+1, T]$, condition 2 of Lemma 1 could be changed by a simpler one.

Lemma 2 Let the following conditions be fulfilled:

1. The delays $\tau_{j}: k-h \leq \tau_{j}(k)<k$ for all $j \in \mathbb{Z}[1, r]$ and $k \in \mathbb{Z}[a+1, T]$.

2. The functions $q, Q, C_{j}: \mathbb{Z}[a+1, T] \rightarrow \mathbb{R}_{+}, j=1,2, \ldots, r$, and

$$
S(k) \equiv q(k)+Q(k)<1 \quad \text { for } k \in \mathbb{Z}[a+1, T] .
$$

3. The function $u: \mathbb{Z}[a+1-h, T] \rightarrow \mathbb{R}_{+}$satisfies inequalities (4).

Then for $k \in \mathbb{Z}[a+1, T]$ the inequality $u(k) \leq K \tilde{\xi}(k)$ holds, where

$$
\tilde{\xi}(k)=\prod_{s=a+1}^{k} \frac{1+\sum_{j=1}^{r} C_{j}(s)}{1-S(s)}+\sum_{s=a+1}^{k}\left[\prod_{\xi=s+1}^{k} \frac{1+\sum_{j=1}^{r} C_{j}(\xi)}{1-S(\xi)}\right] \frac{1}{1-S(s)} .
$$

Corollary 1 Let conditions 1 and 2 of Lemma 1/Lemma 2 be fulfilled, and let the function $u: \mathbb{Z}[a+1-h, T] \rightarrow \mathbb{R}_{+}$satisfy inequalities (4) for $K=0$.

Then $u(k) \leq 0$ for all $k \in \mathbb{Z}[a-h+1, T]$.

\section{Linear delay difference equations with maxima}

In connection with the computer realization of the suggested method, we will give an algorithm for exact solution of the initial value problem for the scalar linear delay-difference equation with 'maxima':

$$
\begin{aligned}
& \Delta u(k-1)= Q(k) u(k)+q(k) \max _{s \in \mathbb{Z}[k-h, k]} u(s) \\
&+\sum_{j=1}^{r} C_{j}(k) u\left(\tau_{j}(k)\right)+P(k), \quad k \in \mathbb{Z}[a+1, T], \\
& u(k)=\psi(k), \quad k \in \mathbb{Z}[a-h+1, a],
\end{aligned}
$$


where $Q, P: \mathbb{Z}[a+1, T] \rightarrow \mathbb{R}, q, C_{j}: \mathbb{Z}[a+1, T] \rightarrow \mathbb{R}_{+}$for $j=1,2, \ldots, r, \psi: \mathbb{Z}[a+1-h, a] \rightarrow \mathbb{R}$ and $\tau_{m}(k): \mathbb{Z}[a+1, T] \rightarrow \mathbb{Z}[a+1-h, T]: k-h \leq \tau_{m}(k) \leq k$ for $m=1,2, \ldots, r$.

We will consider two cases with respect to the type of delays $\tau_{j}$.

Case 1: Let the inequality $\tau_{j}(k)<k$ hold for all $j \in \mathbb{Z}[1, r], k \in \mathbb{Z}[a+1, T]$. Additionally, we assume that inequality (9) is satisfied for all $k \in \mathbb{Z}[a+1, T]$.

Assume that the values $u(k)$ of the unknown solution are obtained for all $k \in \mathbb{Z}[a-h+$ $1, m]$, where $m<T$. Now let $k=m+1$.

Case 1.1: Let the following inequality be satisfied:

$$
\frac{P(k)+\sum_{j=1}^{r} C_{j}(k) u\left(\tau_{j}(k)\right)+u(k-1)}{1-Q(k)-q(k)} \geq \max \{u(k-l): l=1,2, \ldots, h\} .
$$

Thus, $\max _{s \in \mathbb{Z}[k-h, k]} u(s)=u(k)$. Then the unique solution of IVP (11), (12) is

$$
u(k)=\frac{P(k)+\sum_{j=1}^{r} C_{j}(k) u\left(\tau_{j}(k)\right)+u(k-1)}{1-Q(k)-q(k)} .
$$

Case 1.2: Let inequality (13) be not satisfied for all $l \in \mathbb{Z}[1, h]$. Therefore, there exists $m \in \mathbb{Z}[1, h]$ such that $\max _{s \in \mathbb{Z}[k-h, k]} u(s)=u(k-m)$. Then $Q(k) \leq Q(k)+q(k)<1$ and the unique solution of IVP (11), (12) is

$$
u(k)=\frac{P(k)+\sum_{j=1}^{r} C_{j}(k) u\left(\tau_{j}(k)\right)+u(k-1)+q(k) u(k-m)}{1-Q(k)} .
$$

Case 2: Let there exist at least one $j \in \mathbb{Z}[1, r]$ and $k \in \mathbb{Z}[a+1, T]$ such that $\tau_{j}(k)=k$. Additionally, we assume that inequality (5) is satisfied for all $k \in \mathbb{Z}[a+1, T]$.

Assume that the values $u(k)$ of the unknown solution are obtained for all $k \in \mathbb{Z}[a-h+$ $1, m]$, where $m<T$. Now let $k=m+1$.

Let there exist integers $j_{s} \in \mathbb{Z}[1, r]: \tau_{j_{s}}(k)=k$ for $s \in \mathbb{Z}[1, m]$ and $\tau_{j}(k)<k$ for $j \neq j_{s}, s \in$ $\mathbb{Z}[1, m]$. Equation (11) is reduced to

$$
\begin{aligned}
(1 & \left.-Q(k)-\sum_{s=1}^{m} C_{j_{s}}(k)\right) u(k) \\
& =u(k-1)+\sum_{i=1, i \neq j, s \in \mathbb{Z}[1, m]}^{r} C_{i}(k) u\left(\tau_{i}(k)\right)+q(k) \max _{s \in \mathbb{Z}[k-h, k]} u(s)+P(k) .
\end{aligned}
$$

Case 2.1: Let the following inequality be satisfied:

$$
\frac{P(k)+\sum_{i=1, i \neq f j, s \in \mathbb{Z}[1, m]}^{r} C_{i}(k) u\left(\tau_{i}(k)\right)+u(k-1)}{1-Q(k)-\sum_{s=1}^{m} C_{j_{s}}(k)-q(k)} \geq u(k-s), \quad s \in \mathbb{Z}[1, h] .
$$

Therefore, $\max _{s \in \mathbb{Z}[k-h, k]} u(s)=u(k)$, and from (16) we obtain the solution

$$
u(k)=\frac{P(k)+\sum_{i=1, i \neq f j, s \in \mathbb{Z}[1, m]}^{r} C_{i}(k) u\left(\tau_{i}(k)\right)+u(k-1)}{1-Q(k)-\sum_{s=1}^{m} C_{j_{s}}(k)-q(k)} .
$$

Case 2.2: Let inequality (17) be not satisfied for all $s \in \mathbb{Z}[1, h]$. 
Therefore, there exists $m \in \mathbb{Z}[1, h]$ such that $\max _{s \in \mathbb{Z}[k-h, k]} u(s)=u(k-m)$.

Then the unique solution of problem (11), (12) is given by

$$
u(k)=\frac{P(k)+\sum_{i=1, i \neq f j, s \in \mathbb{Z}[1, m]}^{r} C_{i}(k) u\left(\tau_{i}(k)\right)+u(k-1)+q(k) u(k-m)}{1-Q(k)-\sum_{s=1}^{m} C_{j_{s}}(k)} .
$$

\section{Method of quasilinearization}

We will apply the method of quasilinearization to obtain the approximate solution of MBVP (1)-(3).

\section{Theorem 1 Let the following conditions be fulfilled:}

1. The delays $\tau_{j}(k): k-h \leq \tau_{j}(k) \leq k$ for all $j \in \mathbb{Z}[1, r]$ and $k \in \mathbb{Z}[a+1, T]$.

2. The function $f: \mathbb{Z}[a+1, T] \times \mathbb{R}^{r+2} \rightarrow \mathbb{R}$ and for any $k \in \mathbb{Z}[a+1, T]$ and $V \in \Omega_{k}\left(\alpha_{0}, \beta_{0}\right)$, the equality $f(k, V)=F(k, V)-G(k, V)$ holds, where the functions $F(k, V), G(k, V)$ are twice continuously differentiable with respect to any component of $V$ and the following inequalities are valid for $k \in \mathbb{Z}[a+1, T], V \in \Omega_{k}\left(\alpha_{0}, \beta_{0}\right)$ :

$$
\begin{aligned}
& F_{v_{i} v_{j}}(k, V) \geq 0, \quad G_{v_{i} v_{j}}(k, V) \geq 0, \quad \text { for } i, j \in \mathbb{Z}[1, r+2], \\
& F_{v_{j}}\left(k, \Xi\left(\alpha_{0}(k)\right)\right) \geq G_{v_{j}}\left(k, \Xi\left(\beta_{0}(k)\right)\right), \quad j=1,2, \ldots, r+2, \\
& \sum_{j=1}^{r+2}\left[F_{v_{j}}\left(k, \Xi\left(\beta_{0}(k)\right)\right)-G_{v_{j}}\left(k, \Xi\left(\alpha_{0}(k)\right)\right)\right]<1 .
\end{aligned}
$$

3. The function $g \in C^{1}\left(W\left(\alpha_{0}, \beta_{0}\right), \mathbb{R}\right)$ is nondecreasing with respect to all its arguments.

4. The functions $\alpha_{0}, \beta_{0}: \mathbb{Z}[a+1-h, T] \rightarrow \mathbb{R}, \alpha_{0}$ is a lower solution, $\beta_{0}$ is an upper solution of $M B V P(1)-(3)$, and $\alpha_{0}(k) \leq \beta_{0}(k)$ for $k \in \mathbb{Z}[a+1-h, T]$.

Then there exist two sequences of functions $\left\{\alpha_{n}\right\}_{n=0}^{\infty},\left\{\beta_{n}\right\}_{n=0}^{\infty}$ such that

(a) $\alpha_{n}: \mathbb{Z}[a+1-h, T] \rightarrow \mathbb{R}(n=1,2, \ldots)$ are lower solutions of $M B V P(1)-(3)$.

(b) $\beta_{n}: \mathbb{Z}[a+1-h, T] \rightarrow \mathbb{R}(n=1,2, \ldots)$ are upper solutions of $M B V P(1)-(3)$.

(c) $\alpha_{0}(k) \leq \alpha_{1}(k) \leq \cdots \leq \alpha_{n}(k) \leq \cdots \leq \beta_{n}(k) \leq \cdots \leq \beta_{1}(k) \leq \beta_{0}(k)$.

(d) Both sequences are convergent on $\mathbb{Z}[a+1-h, T]$ and their limits $u(k)=\lim _{n \rightarrow \infty} \alpha_{n}(k)$ and $v(k)=\lim _{n \rightarrow \infty} \beta_{n}(k)$ are solutions of $M B V P$ (1)-(3) in $S\left(\alpha_{0}, \beta_{0}\right)$. In the case of uniqueness, both limits coincide with this solution.

(e) The convergence is semi-quadratic, i.e., there exist $\lambda_{i}(k), \mu_{i}(k), v_{i}(k)>0, i=1,2$, such that

$$
\begin{aligned}
& x(k)-\alpha_{n}(k) \leq \lambda_{1}(k)\left\|x-\alpha_{n}\right\|+\mu_{1}(k)\left\|x-\alpha_{n}\right\|^{2}+v_{1}(k)\left\|x-\beta_{n}\right\|^{2}, \\
& \beta_{n}(k)-x(k) \leq \lambda_{2}(k)\left\|x-\beta_{n}\right\|+\mu_{2}(k)\left\|x-\alpha_{n}\right\|^{2}+v_{2}(k)\left\|x-\beta_{n}\right\|^{2} .
\end{aligned}
$$

Proof We will give an algorithm for construction of successive approximations to the exact unknown solution of MBVP (1)-(3).

Assume that the functions $\alpha_{j}(k), \beta_{j}(k): \mathbb{Z}[a+1-h, T] \rightarrow \mathbb{R}, j=1,2, \ldots, n$, are constructed so that the following conditions are satisfied:

(H1) $\alpha_{j}(k) \geq \alpha_{j-1}(k)$ and $\beta_{j}(k) \leq \beta_{j-1}(k)$ for $k \in \mathbb{Z}[a+1-h, T]$;

(H2) $\alpha_{j}(k) \leq \beta_{j}(k)$ for $k \in \mathbb{Z}[a+1-h, T]$;

(H3) $\alpha_{j}(k) \leq \varphi(k) \leq \beta_{j}(k)$ for $k \in \mathbb{Z}[a+1-h, a-1]$; 
(H4) functions $\alpha_{j}, \beta_{j}$ are lower and upper solutions of MBVP (1)-(3), respectively. Consider both initial value problems (IVP) for the linear difference equations with 'maxima':

$$
\begin{aligned}
& \begin{aligned}
\Delta x(k-1)= & Q_{n}(k) x(k)+\sum_{m=1}^{r} C_{m}^{(n)}(k) x\left(\tau_{m}(k)\right)+q_{n}(k) \max _{s \in \mathbb{Z}[k-h, k]} x(s) \\
& +P_{n}\left(k, \alpha_{n}(k)\right), \quad k \in \mathbb{Z}[a+1, T], \\
x(k)= & \varphi(k)-k_{n} L_{n}, \quad k \in \mathbb{Z}[a+1-h, a-1], \\
x(a)= & g\left(\chi\left(\alpha_{n}(T)\right)\right),
\end{aligned}
\end{aligned}
$$

and

$$
\begin{aligned}
& \Delta x(k-1)=Q_{n}(k) x(k)+\sum_{m=1}^{r} C_{m}^{(n)}(k) x\left(\tau_{m}(k)\right)+q_{n}(k) \max _{s \in \mathbb{Z}[k-h, k]} x(s) \\
& +P_{n}\left(k, \beta_{n}(k)\right), \quad k \in \mathbb{Z}[a+1, T], \\
& x(k)=\varphi(k)+p_{n} M_{n}, \quad k \in \mathbb{Z}[a+1-h, a-1], \\
& x(a)=g\left(\chi\left(\beta_{n}(T)\right)\right),
\end{aligned}
$$

where

$$
\begin{aligned}
& L_{n}=\min _{s \in \mathbb{Z}[a-h+1, a-1]}\left[\varphi(s)-\alpha_{n}(s)\right], \quad M_{n}=\min _{s \in \mathbb{Z}[a-h+1, a-1]}\left[\beta_{n}(s)-\varphi(s)\right], \\
& k_{n}=\min \left\{L_{n}, \frac{1}{2^{n}}\right\}, \quad p_{n}=\min \left\{M_{n}, \frac{1}{2^{n}}\right\},
\end{aligned}
$$

and $P_{n}, Q_{n}, q_{n}, C_{m}: \mathbb{Z}[a+1, T] \rightarrow \mathbb{R}, m=1,2, \ldots, r$, are defined by

$$
\begin{aligned}
& P_{n}(k, u(k))=f(k, \Xi(u(k)))-Q_{n}(k) u(k)-\sum_{m=1}^{r} C_{m}^{(n)}(k) u\left(\tau_{m}(k)\right)-q_{n}(k) \max _{s \in \mathbb{Z}[k-h, k]} u(s), \\
& Q_{n}(k)=F_{v_{1}}\left(k, \Xi\left(\alpha_{n}(k)\right)\right)-G_{v_{1}}\left(k, \Xi\left(\beta_{n}(k)\right)\right) \geq 0, \\
& q_{n}(k)=F_{v_{r+2}}\left(k, \Xi\left(\alpha_{n}(k)\right)\right)-G_{v_{r+2}}\left(k, \Xi\left(\beta_{n}(k)\right)\right) \geq 0, \\
& C_{m-1}^{(n)}(k)=F_{v_{m}}\left(k, \Xi\left(\alpha_{n}(k)\right)\right)-G_{v_{m}}\left(k, \Xi\left(\beta_{n}(k)\right)\right), \quad m=2,3, \ldots, r+1 .
\end{aligned}
$$

From inequalities (20) and $\alpha_{n}(k) \geq \alpha_{0}(k), \beta_{n}(k) \leq \beta_{0}(k), k \in \mathbb{Z}[a+1-h, T]$, it follows that $q_{n}(k) \geq 0$. From inequalities (20), (22) we get that the functions $Q_{n}, q_{n}, C_{m-1}^{(n)}$ satisfy (5) for $k \in \mathbb{Z}[a+1, T]$. IVPs (23), (24) and (25), (26) have unique solutions $\alpha_{n+1}(k)$ and $\beta_{n+1}(k)$, respectively, which are defined on the interval $\mathbb{Z}[a+1-h, T]$.

From condition (H3), for $j=n$, the constants $L_{n}, M_{n}, k_{n}, p_{n} \geq 0$ follow.

Define a function $p_{1}: \mathbb{Z}[a+1-h, T] \rightarrow \mathbb{R}$ by $p_{1}(k)=\alpha_{n}(k)-\alpha_{n+1}(k)$.

Let $k \in \mathbb{Z}[a+1-h, a-1]$. From (24) we get $\varphi(k)-\alpha_{n}(k) \geq L_{n}$ and

$$
p_{1}(k) \leq-L_{n}+k_{n} L_{n}=\left(-1+k_{n}\right) L_{n} \leq 0 \quad \text { for } k \in \mathbb{Z}[a+1-h, a-1]
$$


Now, let $k=a$. Then from (H3) for $j=n$ we get

$$
p_{1}(a)=\alpha_{n}(a)-g\left(\chi\left(\alpha_{n}(T)\right)\right) \leq 0 .
$$

Let $k \in \mathbb{Z}[a+1, T]$. From the choice of the function $\alpha_{n}$ and equation (23) for the function $\alpha_{n+1}$, we get

$$
\Delta p_{1}(k-1) \leq Q_{n}(k) p_{1}(k)+\sum_{j=1}^{r} C_{j}^{(n)}(k) p_{1}\left(\tau_{j}(k)\right)+q_{n}(k) \max _{s \in \mathbb{Z}[k-h, k]} p_{1}(s) .
$$

According to Corollary 1, from inequalities (27)-(29) it follows that $p_{1}(k) \leq 0$ for $k \in$ $\mathbb{Z}[a+1-h, T]$, i.e., $\alpha_{n}(k) \leq \alpha_{n+1}(k)$ for $k \in \mathbb{Z}[a+1-h, T]$.

Similarly, we prove $\beta_{n}(k) \geq \beta_{n+1}(k)$ for $k \in \mathbb{Z}[a+1-h, T]$, i.e., condition (H1) is satisfied for $j=n+1$.

Now, we will prove that the function $\alpha_{n+1}$ is a lower solution of MBVP (1)-(3).

Let $k \in \mathbb{Z}[a+1-h, a-1]$. From (24) we get $\alpha_{n+1}(k)=\varphi(k)-k_{n} L_{n} \leq \varphi(k)$.

Let $k=a$. From the proved above and condition 3 of Theorem 1, the inequality $\alpha_{n+1}(a)=$ $g\left(\chi\left(\alpha_{n}(T)\right)\right) \leq g\left(\chi\left(\alpha_{n+1}(T)\right)\right)$ holds.

Let $k \in \mathbb{Z}[a+1, T]$. Then we obtain

$$
\begin{aligned}
\Delta \alpha_{n+1}(k-1)= & \left(F_{v_{1}}\left(k, \Xi\left(\alpha_{n}(k)\right)\right)-G_{v_{1}}\left(k, \Xi\left(\beta_{n}(k)\right)\right)\left(\alpha_{n+1}(k)-\alpha_{n}(k)\right)\right. \\
& +\sum_{j=1}^{r} C_{j}^{(n)}(k)\left(\alpha_{n+1}\left(\left(\tau_{j}(k)\right)\right)-\alpha_{n}\left(\left(\tau_{j}(k)\right)\right)\right) \\
& +\left(F_{v_{r+2}}\left(k, \Xi\left(\alpha_{n}(k)\right)\right)-G_{v_{r+2}}\left(k, \Xi\left(\beta_{n}(k)\right)\right)\right) \\
& \times\left(\max _{s \in \mathbb{Z}[k-h, k]} \alpha_{n+1}(s)-\max _{s \in \mathbb{Z}[k-h, k]} \alpha_{n}(s)\right) \\
& +\left(f\left(k, \Xi\left(\alpha_{n}(k)\right)\right)-f\left(k, \Xi\left(\alpha_{n+1}(k)\right)\right)\right)-f\left(k, \Xi\left(\alpha_{n+1}(k)\right)\right) \\
\leq & f\left(k, \Xi\left(\alpha_{n+1}(k)\right)\right) .
\end{aligned}
$$

Similarly, we prove that $\beta_{n+1}(k)$ is an upper solution of MBVP (1)-(3).

Now, we will prove (H2) for $j=n+1$. Define a function $p_{3}: \mathbb{Z}[a+1-h, T] \rightarrow \mathbb{R}$ by the equality $p_{3}(k)=\alpha_{n+1}(k)-\beta_{n+1}(k)$.

Let $k \in \mathbb{Z}[a+1-h, a-1]$. Then $p_{3}(k)=\varphi(k)-k_{n} L_{n}-\varphi(k)-p_{n} M_{n} \leq 0$.

Also, from condition 3 of Theorem 1 and (H2) for $j=n$, we get $p_{3}(a)=g\left(\chi\left(\alpha_{n}(T)\right)\right)-$ $g\left(\chi\left(\beta_{n}(T)\right)\right) \leq 0$.

Now, let $k \in \mathbb{Z}[a+1, T]$. Then for the function $p_{3}(k)$ we get

$$
\begin{aligned}
\Delta p_{3}(k-1)= & f\left(k, \Xi\left(\alpha_{n}(k)\right)\right)-f\left(k, \Xi\left(\beta_{n}(k)\right)\right) \\
& -Q_{n}(k)\left[\beta_{n+1}(k)-\beta_{n}(k)\right]+\sum_{j=1}^{r} C_{j}^{(n)}(k)\left[\alpha_{n+1}\left(\tau_{j}(k)\right)-\alpha_{n}\left(\tau_{j}(k)\right)\right] \\
& +Q_{n}(k)\left[\alpha_{n+1}(k)-\alpha_{n}(k)\right]-\sum_{j=1}^{r} C_{j}^{(n)}(k)\left[\beta_{n+1}\left(\tau_{j}(k)\right)-\beta_{n}\left(\tau_{j}(k)\right)\right]
\end{aligned}
$$




$$
\begin{aligned}
& +q_{n}(k)\left[\max _{s \in \mathbb{Z}[k-h, k]} \alpha_{n+1}(s)-\max _{s \in \mathbb{Z}[k-h, k]} \alpha_{n}(s)\right] \\
& -q_{n}(k)\left[\max _{s \in \mathbb{Z}[k-h, k]} \beta_{n+1}(s)-\max _{s \in \mathbb{Z}[k-h, k]} \beta_{n}(s)\right] \\
\leq & Q_{n}(k) p_{3}(k)+\sum_{j=1}^{r} C_{j}^{(n)}(k) p_{3}\left(\tau_{j}(k)\right)+q_{n}(k) \max _{s \in \mathbb{Z}[k-h, k]} p_{3}(s) .
\end{aligned}
$$

According to Corollary 1 , the inequality $p_{3}(k) \leq 0$ holds for $k \in \mathbb{Z}[a+1-h, T]$, i.e., $\alpha_{n+1}(k) \leq \beta_{n+1}(k)$ for $k \in \mathbb{Z}[a+1-h, T]$.

Therefore, $\alpha_{n+1}, \beta_{n+1} \in S\left(\alpha_{0}, \beta_{0}\right)$.

For any fixed $k \in \mathbb{Z}[a+1-h, T]$, the sequences $\left\{\alpha_{n}\right\}_{n=0}^{\infty}$ and $\left\{\beta_{n}\right\}_{n=0}^{\infty}$ are monotone nondecreasing and monotone nonincreasing, respectively, and they are bounded by the functions $\alpha_{0}$ and $\beta_{0}$. Therefore, both sequences are convergent on $\mathbb{Z}[a+1-h, T]$, i.e., there exist functions $V, W: \mathbb{Z}[a+1-h, T] \rightarrow \mathbb{R}$ such that

$$
\lim _{n \rightarrow \infty} \alpha_{n}(k)=V(k) \quad \text { and } \quad \lim _{n \rightarrow \infty} \beta_{n}(k)=W(k) \quad \text { for } k \in \mathbb{Z}[a+1-h, T] .
$$

From claim (c) of Theorem 1 it follows that $V, W \in S\left(\alpha_{0}, \beta_{0}\right)$. Taking limits as $n \rightarrow \infty$ in IVPs (23), (24) and (25), (26), we obtain that $V$ and $W$ are solutions of MBVP (1)-(3) in $S\left(\alpha_{0}, \beta_{0}\right)$.

Now, we will study the power of convergence of the sequences of functions $\left\{\alpha_{n}\right\}_{n=0}^{\infty}$ and $\left\{\beta_{n}\right\}_{n=0}^{\infty}$.

Define the functions $\tilde{A}_{n+1}, \tilde{B}_{n+1}: \mathbb{Z}[a+1-h, T] \rightarrow \mathbb{R}_{+}, n=0,1, \ldots$, by

$$
\tilde{A}_{n+1}(k)=x(k)-\alpha_{n+1}(k), \quad \tilde{B}_{n+1}(k)=\beta_{n+1}(k)-x(k) .
$$

Let $k \in \mathbb{Z}[a+1-h, a-1]$. From (24) and the choice of $\alpha_{n}$ we get

$$
\tilde{A}_{n+1}(k)=k_{n} L_{n} \leq L_{n}^{2} \leq\left\|\tilde{A}_{n}\right\|^{2} .
$$

Also, from condition 3 it follows that there exists a constant $C>0$ such that

$$
\begin{aligned}
\tilde{A}_{n+1}(a) & =g(\chi(x(T)))-g\left(\chi\left(\alpha_{n}(T)\right)\right) \\
& \leq g_{v_{p+1}}(\chi(x(T)))\left(x(T)-\alpha_{n}(T)\right)+\sum_{j=1}^{p} g_{v_{j}}(\chi(x(T)))\left(x\left(T-\lambda_{j}\right)-\alpha_{n}\left(T-\lambda_{j}\right)\right) \\
& \leq C\left\|\tilde{A}_{n}\right\| .
\end{aligned}
$$

Let $k \in \mathbb{Z}[a+1, T]$. According to the definitions of the functions $\tilde{A}_{n+1}(k), \alpha_{n+1}(k)$ and condition 2 of Theorem 1 , we get

$$
\begin{aligned}
& \Delta \tilde{A}_{n+1}(k-1) \\
& \leq Q_{n}(k) \tilde{A}_{n+1}(k)+q_{n}(k) \max _{s \in \mathbb{Z}[k-h, k]} \tilde{A}_{n+1}(s)+\sum_{m=1}^{r} C_{m}^{(n)}(k) \tilde{A}_{n+1}\left(\tau_{m}(k)\right) \\
& \quad+\left[F_{v_{1}}(k, \Xi(x(k)))-G_{v_{1}}\left(k, \Xi\left(\alpha_{n}(k)\right)\right)-Q_{n}(k)\right] \tilde{A}_{n}(k)
\end{aligned}
$$




$$
\begin{aligned}
& +\left[F_{v_{r+2}}(k, \Xi(x(k)))-G_{v_{r+2}}\left(k, \Xi\left(\alpha_{n}(k)\right)\right)-q_{n}(k)\right] \\
& \times\left(\max _{s \in \mathbb{Z}[k-h, k]} x(s)-\max _{s \in \mathbb{Z}[k-h, k]} \alpha_{n}(s)\right)-\sum_{m=1}^{r} C_{m}^{(n)}(k) \tilde{A}_{n}\left(\tau_{m}(k)\right) .
\end{aligned}
$$

Also, the following inequalities are valid:

$$
\begin{aligned}
& \tilde{A}_{n}(k)\left[\beta_{n}(k)-\alpha_{n}(k)\right] \leq \tilde{A}_{n}(k)\left[\tilde{A}_{n}(k)+\tilde{B}_{n}(k)\right] \leq \frac{3}{2} \tilde{A}_{n}^{2}(k)+\frac{1}{2} \tilde{B}_{n}^{2}(k), \\
& \tilde{A}_{n}(k)\left[\max _{s \in \mathbb{Z}[k-h, k]} \beta_{n}(s)-\max _{s \in \mathbb{Z}[k-h, k]} \alpha_{n}(s)\right] \leq \frac{3}{2}\left\|\tilde{A}_{n}\right\|^{2}+\frac{1}{2}\left\|\tilde{B}_{n}\right\|^{2}, \\
& \tilde{A}_{n}(k)\left[\max _{s \in \mathbb{Z}[k-h, k]} \beta_{n}(s)-\max _{s \in \mathbb{Z}[k-h, k]} \alpha_{n}(s)\right] \leq \frac{3}{2}\left\|\tilde{A}_{n}\right\|^{2}+\frac{1}{2}\left\|\tilde{B}_{n}\right\|^{2}, \\
& \max _{s \in \mathbb{Z}[k-h, k]} \tilde{A}_{n}(s)\left[\beta_{n}(k)-\alpha_{n}(k)\right] \leq \frac{3}{2}\left\|\tilde{A}_{n}\right\|^{2}+\frac{1}{2}\left\|\tilde{B}_{n}\right\|^{2}, \\
& \max _{s \in \mathbb{Z}[k-h, k]} \tilde{A}_{n}(s)\left[\max _{s \in \mathbb{Z}[k-h, k]} \beta_{n}(s)-\max _{s \in \mathbb{Z}[k-h, k]} \alpha_{n}(s)\right] \leq \frac{3}{2}\left\|\tilde{A}_{n}\right\|^{2}+\frac{1}{2}\left\|\tilde{B}_{n}\right\|^{2} .
\end{aligned}
$$

Applying the mean value theorem, condition 2 of Theorem 1, inequalities (33), (34) and some simple calculations, we obtain that there exist constants $M_{k}, S_{k}>0$ such that

$$
\begin{aligned}
\Delta \tilde{A}_{n+1}(k-1) \leq & Q_{n}(k) \tilde{A}_{n+1}(k)+q_{n}(k) \max _{s \in \mathbb{Z}[k-h, k]} \tilde{A}_{n+1}(s) \\
& +\sum_{j=1}^{r} C_{j}^{(n)}(k) \tilde{A}_{n+1}\left(\tau_{j}(k)\right)+M_{k}\left\|\tilde{A}_{n}\right\|^{2}+S_{k}\left\|\tilde{B}_{n}\right\|^{2} .
\end{aligned}
$$

Let $M=\max \left\{1, \max _{k \in \mathbb{Z}[a+1, T]} M_{k}\right\}$ and $S=\max \left\{1, \max _{k \in \mathbb{Z}[a+1, T]} S_{k}\right\}$.

According to Lemma 1, for $u=\tilde{A}_{n+1}, K=C\left\|\tilde{A}_{n}\right\|+M\left\|\tilde{A}_{n}\right\|^{2}+S\left\|\tilde{B}_{n}\right\|^{2}$ from inequalities (31), (32), (35) it follows that

$$
\tilde{A}_{n+1}(k) \leq\left(C\left\|\tilde{A}_{n}\right\|+M\left\|\tilde{A}_{n}\right\|^{2}+S\left\|\tilde{B}_{n}\right\|^{2}\right) \theta(k)
$$

where $\theta(k)$ is defined by (6) for $\tilde{S}(k)=Q_{n}(k)+\sum_{j=1}^{r} C_{j}^{(n)}(k)+q_{n}(k)$, i.e., the sequence $\left\{\alpha_{n}\right\}_{n=0}^{\infty}$ semi-quadratically converges to the exact solution of (1)-(3).

Similarly, we prove the semi-quadratic convergence of $\left\{\beta_{n}\right\}_{n=0}^{\infty}$.

Theorem 2 Let the delays $\tau_{j}(k): k-h \leq \tau_{j}(k)<k$ for all $j \in \mathbb{Z}[1, r]$ and $k \in \mathbb{Z}[a+1, T]$ and conditions 2, 3, 4 of Theorem 1 be satisfied, where the inequalities (21) and (22) are replaced by

$$
\begin{aligned}
& F_{v_{j}}\left(k, \Xi\left(\alpha_{0}(k)\right)\right) \geq G_{v_{j}}\left(k, \Xi\left(\beta_{0}(k)\right)\right) \text { for } j=1 \text { and } j=r+2, \\
& F_{v_{1}}\left(k, \Xi\left(\beta_{0}(k)\right)\right)+F_{v_{r+2}}\left(k, \Xi\left(\beta_{0}(k)\right)\right) \\
& \quad-G_{v_{1}}\left(k, \Xi\left(\alpha_{0}(k)\right)\right)-G_{v_{r+2}}\left(k, \Xi\left(\alpha_{0}(k)\right)\right)<1 .
\end{aligned}
$$




\section{Application}

Now we will give an example of a generalized difference equation to illustrate the advantage of both the introducing delay functions $\tau_{r}$ in the equation and the suggested above scheme for approximate obtaining of a solution.

Consider the MBVP for the nonlinear difference equation with 'maxima'

$$
\Delta u(k-1)=\frac{2^{k-1}}{2^{k+1}-u(k)}+\frac{2^{k-2}}{2^{k+1}-\max _{s \in \mathbb{Z}[k-3, k]} u(s)}+\frac{2^{k-1}}{2^{k+1}-u(\tau(k))},
$$

for $k \in \mathbb{Z}[3,15]$,

$$
u(0)=0, \quad u(1)=0.5, \quad u(2)=(1.1)^{0.1 u(15)}
$$

where $\tau(k)=k-[\sqrt{k}], k \in \mathbb{Z}[3,15]$, and $[s]$ denotes the integer part of the real number $s$, i.e.,

$$
\tau(k)= \begin{cases}k-1, & k=3 \\ k-2, & k=4,5,6,7,8, \\ k-3, & k=9,10,11,12,13,14,15\end{cases}
$$

$\operatorname{MBVP}(39)$ is of type (1)-(3), where $h=3, a=2, T=15, g(u)=(1.1)^{0.1 u}, \varphi(0)=0, \varphi(1)=0.5$, $f \equiv F, G \equiv 0, F\left(k, v_{1}, v_{2}, v_{3}\right)=\frac{2^{k-1}}{2^{k+1}-v_{1}}+\frac{2^{k-2}}{2^{k+1}-v_{2}}+\frac{2^{k-1}}{2^{k+1}-v_{3}}$.

The functions $\alpha_{0}(k)=-k$ and $\beta_{0}(k)=k, k \in \mathbb{Z}[0,15]$ are lower and upper solutions of (39). Inequalities (37), (38) and condition 3 of Theorem 1 are satisfied. According to Theorem 2, MBVP (39) has a solution, and we will obtain it as a limit of two sequences of successive approximations.

The approximation $\alpha_{n}$ is a solution of IVP (23), (24) which is reduced to

$$
\begin{aligned}
\Delta \alpha_{n}(k-1)= & \frac{2^{k-1} \alpha_{n}(k)}{\left(2^{k+1}-\alpha_{n-1}(k)\right)^{2}}+\frac{2^{k-1}}{\left(2^{k+1}-\alpha_{n-1}(\tau(k))\right)^{2}} \alpha_{n}(\tau(k)) \\
& +\frac{2^{k-2}}{\left(2^{k+1}-\max _{s \in \mathbb{Z}[k-3, k]} \alpha_{n-1}(s)\right)^{2}} \max _{s \in \mathbb{Z}[k-3, k]} \alpha_{n}(s)+P\left(k, \alpha_{n-1}\right),
\end{aligned}
$$

$$
k \in \mathbb{Z}[3,15]
$$

$$
\alpha_{n}(0)=-k_{n-1} L_{n-1}, \quad \alpha_{n}(1)=0.5-k_{n-1} L_{n-1}, \quad \alpha_{n}(2)=(1.1)^{0.1 \alpha_{n-1}(15)},
$$

and the approximation $\beta_{n}$ is a solution of IVP (25), (26) which is reduced to

$$
\begin{aligned}
\Delta \beta_{n}(k-1)= & \frac{2^{k-1} \beta_{n}(k)}{\left(2^{k+1}-\beta_{n-1}(k)\right)^{2}}+\frac{2^{k-1}}{\left(2^{k+1}-\beta_{n-1}(\tau(k))\right)^{2}} \beta_{n}(\tau(k)) \\
& +\frac{2^{k-2}}{\left(2^{k+1}-\max _{s \in \mathbb{Z}[k-3, k]} \beta_{n-1}(s)\right)^{2}} \max _{s \in \mathbb{Z}[k-3, k]} \beta_{n}(s)+P\left(k, \beta_{n-1}\right),
\end{aligned}
$$

$$
k \in \mathbb{Z}[3,15]
$$

$$
\beta_{n}(0)=p_{n-1} M_{n-1}, \quad \beta_{n}(1)=0.5+p_{n-1} M_{n-1}, \quad \beta_{n}(2)=(1.1)^{0.1 \beta_{n-1}(15)},
$$


Table 1 Values of successive lower approximations $\alpha_{n}(k)$ and upper approximations $\beta_{n}(k)$, $n=0,1,2,3,4,5,6$

\begin{tabular}{|c|c|c|c|c|c|c|c|c|}
\hline $\bar{k}$ & 0 & 1 & 2 & 3 & 4 & $\ldots$ & 14 & 15 \\
\hline$\overline{\beta_{0}(k)}$ & 0 & 1.0 & 2.000000 & 3.000000 & 4.000000 & $\ldots$ & 14.000000 & 15.000000 \\
\hline$\beta_{1}(k)$ & 0 & 0.5 & 1.153690 & 1.778772 & 2.413602 & $\ldots$ & 8.676488 & 9.301502 \\
\hline$\beta_{2}(k)$ & 0 & 0.5 & 1.092701 & 1.751804 & 2.387379 & $\ldots$ & 8.663076 & 9.287101 \\
\hline$\beta_{3}(k)$ & 0 & 0.5 & 1.092551 & 1.742376 & 2.388160 & $\ldots$ & 8.662967 & 9.287992 \\
\hline$\beta_{4}(k)$ & 0 & 0.5 & 1.092561 & 1.742380 & 2.388161 & $\ldots$ & 8.662964 & 9.287989 \\
\hline$u(k)$ & 0 & 0.5 & 1.092561 & 1.742380 & 2.388161 & $\ldots$ & 8.662964 & 9.287989 \\
\hline$\alpha_{6}(k)$ & 0 & 0.5 & 1.092561 & 1.742380 & 2.388161 & $\ldots$ & 8.662964 & 9.287989 \\
\hline$\alpha_{5}(k)$ & 0 & 0.5 & 1.092561 & 1.742379 & 2.388161 & $\ldots$ & 8.662964 & 9.287988 \\
\hline$\alpha_{4}(k)$ & 0 & 0.5 & 1.092561 & 1.742379 & 2.388160 & $\ldots$ & 8.662963 & 9.287988 \\
\hline$\alpha_{3}(k)$ & 0 & 0.5 & 1.092597 & 1.742418 & 2.388160 & $\ldots$ & 8.662959 & 9.288005 \\
\hline$\alpha_{2}(k)$ & 0 & 0.5 & 1.090370 & 1.743462 & 2.390556 & $\ldots$ & 8.666457 & 9.281482 \\
\hline$\alpha_{1}(k)$ & 0 & 0.5 & 0.866784 & 1.512146 & 2.159955 & $\ldots$ & 8.452380 & 9.077444 \\
\hline$\alpha_{0}(k)$ & 0 & -1.0 & -2.000000 & -3.000000 & -4.00000 & $\ldots$ & -14.000000 & -15.000000 \\
\hline
\end{tabular}

where $L_{n-1}=\min \left\{-\alpha_{n-1}(0), 0.5-\alpha_{n-1}(1)\right\}, M_{n-1}=\min \left\{\beta_{n-1}(0), \beta_{n-1}(1)-0.5\right\}, k_{n-1}=$ $\min \left\{L_{n-1}, \frac{1}{2^{n-1}}\right\}, p_{n-1}=\min \left\{M_{n-1}, \frac{1}{2^{n-1}}\right\}$ and

$$
\begin{aligned}
P(k, u)= & \frac{2^{k-2}}{2^{k+1}-\max _{s \in \mathbb{Z}[k-3, k]} u(s)}\left(1-\frac{\max _{s \in \mathbb{Z}[k-3, k]} u(s)}{2^{k+1}-\max _{s \in \mathbb{Z}[k-3, k]} u(s)}\right) \\
& +\frac{2^{k-1}}{2^{k+1}-u(k)}\left(1-\frac{u(k)}{2^{k+1}-u(k)}\right)+\frac{2^{k-1}}{2^{k+1}-u(\tau(k))}\left(1-\frac{u(\tau(k))}{2^{k+1}-u(\tau(k))}\right) .
\end{aligned}
$$

IVPs (40) and (41) are solved by a computer realization of the algorithm given in Section 5 , and the results are given in Table 1 . The obtained numerical results demonstrate the rapid monotonic convergence of both sequences.

\section{Competing interests}

The authors declare that they have no competing interests.

\section{Authors' contributions}

All authors contributed equally in the preparation of this article. Both authors read and approved the final manuscript.

\section{Acknowledgements}

Research was partially supported by Fund Scientific Research MU13FMI002, Plovdiv University.

Received: 29 October 2013 Accepted: 27 January 2014 Published: 31 Mar 2014

\section{References}

1. Wang, $Q$, Perc, M, Duan, Z, Chen, G: Delay-induced multiple stochastic resonances on scale-free neuronal networks. Chaos 19,023112 (2009). doi:10.1063/1.3133126

2. Perc, M: Spatial coherence resonance in neuronal media with discrete local dynamics. Chaos Solitons Fractals 31, 64-69 (2007)

3. Ferraro, T, de Candia, A, Gamba, A, Coniglio, A: Synchronization transitions on small-world neuronal networks: effects of information transmission delay and rewiring probability. EPL 83, 50008 (2008). doi:10.1209/0295-5075/83/50009

4. Wang, Q, Perc, M, Duan, Z, Chen, G: Synchronization transitions on scale-free neuronal networks due to finite information transmission delays. Phys. Rev. E 80, 026206 (2009)

5. Gelisken, A, Sinar, G, Kurbanli, A: On the asymptotic behavior and periodic nature of a difference equation with maximum. Comput. Math. Appl. 59(2), 898-902 (2010)

6. Touafek, N, Halim, Y: On max type difference equations: expressions of solutions. Int. J. Nonlinear Sci. 11(4), 396-402 (2011)

7. Yang, X, Liao, X, Li, C: On a difference equation with maximum. Appl. Math. Comput. 181(1), 1-5 (2006)

8. Atici, FM, Cabada, A, Ferreiro, J: First order difference equations with maxima and nonlinear functional boundary value conditions. J. Differ. Equ. Appl. 12(6), 565-576 (2006)

9. Tisdell, C: On first-order discrete boundary value problems. J. Differ. Equ. Appl. 12, 1213-1223 (2006)

10. Agarwal, R, Cabada, A, Otero-Espinar, V: Existence and uniqueness results for $n$-th order nonlinear difference equations in presence of lower and upper solutions. Arch. Inequal. Appl. 1, 421-432 (2003) 
11. Jankowski, T: First-order functional difference equations with nonlinear boundary value problems. Comput. Math. Appl. 59, 1937-1943 (2010)

12. Cabada, A: The method of lower and upper solutions for periodic and anti periodic difference equations. Electron. Trans. Numer. Anal. 27, 13-25 (2007)

13. Cabada, A, Otero-Espinar, V, Pouso, V: Existence and approximations of solutions of first order discontinuous difference equations with nonlinear global conditions in the presence of lower and upper solutions. Comput. Math. Appl. 39, 21-33 (2000)

14. Hristova, S, Golev, A, Stefanova, K: Quasilinearization of the initial value problem for difference equations with maxima. J. Appl. Math. 2012, Article ID 159031 (2012). doi:10.1155/2012/159031

15. Atici, FM, Cabada, A, Ferreiro, J: Existence and comparison results for first order periodic implicit difference equations with maxima. J. Differ. Equ. Appl. 8(4), 357-369 (2002)

16. Agarwal, R, Hristova, S: Quasilinearization for initial value problems involving differential equations with "maxima". Math. Comput. Model. 55(9-10), 2096-2105 (2012)

17. Bainov, D, Hristova, S: Differential Equations with Maxima. Taylor \& Francis, CRC Press, London (2011)

18. Golev, A, Hrisova, S, Rahnev, A: An algorithm for approximate solving of differential equations with "maxima". Comput. Math. Appl. 60, 2771-2778 (2010)

10.1186/1029-242X-2014-132

Cite this article as: Hristova and Golev: Quasilinearization for nonlinear boundary value problems for delay-type difference equations with maxima. Journal of Inequalities and Applications 2014, 2014:132

\section{Submit your manuscript to a SpringerOpen ${ }^{0}$ journal and benefit from:}

- Convenient online submission

- Rigorous peer review

- Immediate publication on acceptance

Open access: articles freely available online

- High visibility within the field

- Retaining the copyright to your article 